\title{
Nápoles, ciudad democrática: la construcción del centro histórico como un espacio público
}

UGO Rossi*

\begin{abstract}
This paper analyses the process of urban regeneration that has occurred in the historic centre of Naples over the last decade. My thesis is that this process has arisen from a mixture of differentiated forms of collective action and social mobilisation. In particular, I want to put forward a double-level framework of analysis which takes into consideration two perspectives on the process of urban change: from above and from below. In my case-study, the former has been led by the local political elites and the judges, while the latter by the civil society and the social movements. This sheds light on the current complexity of urban change processes and on the multiplicity of sources of social power at the urban scale. Indeed, the very purpose of this paper is to discuss the idea of a multiplex city and to propose an active approach to post-modern urbanism, which challenges pessimistic visions on the future of cities.
\end{abstract}

Keywords: post-modern urbanism, multiplex city, social movements, civil society, reformist urban planning, public space, historic centre of Naples.

\section{Resumen}

El presente artículo analiza el proceso de regeneración urbana que ha tenido lugar en el centro histórico de Nápoles en la última década. Mi tesis es que dicho proceso ha ocurrido gracias una mezcla de formas diferenciadas de acción colectiva y movilización social. En particular, presento un marco de análisis que considera dos perspectivas en el proceso de cambio urbano: desde arriba y desde abajo. En mi estudio de caso, la primera perspectiva se ha dado por las élites políticas locales y los jueces, mientras que la segunda por la sociedad civil y los movimientos sociales. Este análisis aborda la complejidad actual de los procesos de cambio urbano y la multiplicidad de fuentes de poder social a escala urbana. El propósito de este artículo es discutir la idea de ciudad múltiple y proponer un enfoque activo al urbanismo postmoderno, el cual se enfrenta a las visiones pesimistas acerca del futuro de las ciudades.

Palabras clave: urbanismo postmoderno, ciudad múltiple, movimientos sociales, planeación urbana reformista, espacio público, centro histórico de Nápoles.

*Università di Napoli “L’Orientale”, Italia. Correo-e: urossi@iuo.it 


\section{Introducción ${ }^{1}$}

En los últimos años, un creciente número de investigadores ha comenzado a reconsiderar de manera positiva el futuro de las ciudades, tomando en cuenta asuntos tales como la justicia social, la democracia sustantiva y el compromiso cívico (Dematteis et al. 1999; Holston, 1999; Friedmann, 2000; Harvey, 2000; Amin y Thrift, 2002). Este re-descubrimiento de temas que estuvieron de moda en la década de 1970 puede ser entendido como una reacción al enfoque centrado en el mercado acerca de la cuestión urbana, el cual se volvió una fuerte influencia en los debates políticos y científicos después del vuelco neoliberal de la década de 1980. En este sentido, se podría argumentar que estas últimas tendencias en la investigación urbana reflejan la manera en la cual los urbanistas críticos y progresivos han comenzado a abandonar los sentimientos de derrota y resignación que por largo tiempo han prevalecido en este campo.

Algunas señales de esta nueva actitud pueden encontrarse en la literatura sobre cambio urbano. Así pues, mientras la teoría actual se ha centrado por mucho tiempo en la regeneración urbana y regional con niveles cada vez más altos de competitividad, hoy en día se está gestando un nuevo enfoque del cambio urbano. Los estudios urbanos, de hecho, están interesados cada vez más en el mantenimiento político y social de los procesos de cambio urbano, enfocándose particularmente en las cuestiones de la democracia y la esfera pública, en la formación de nuevas demandas y de ciudadanía activa (García, 1996; Imrie et al., 1996; Beauregard y Body-Gendrot, 1999).

Basado en estas premisas, este artículo intenta abordar la cuestión de la ciudad democrática desarrollando empíricamente la idea de "ciudad múltiple" (Amin y Graham, 1997; véase también Thrift, 2001). Haciendo énfasis en la heterogeneidad económica y social y los bienes institucionales sobre los cuales la vida urbana contemporánea se fundamenta, este enfoque investiga la multiplicidad de las "fuentes de poder social" (Mann, 1993) que nutren a la democracia urbana.

\footnotetext{
${ }^{1}$ Una versión preliminar de este artículo fue presentada en la Iv Conferencia Europea sobre Estudios Urbanos y Regionales: “(Re)placing Europe: economies, territories and identities”, Barcelona, julio de 2002. Estoy agradecido con la Mesa de Redacción de Economía, Sociedad y Territorio por la traducción de esta artículo al español, y también quiero agradecer a Rosario Rogel y a dos árbitros anónimos por su útil respaldo. Las omisiones y errores son de mi entera responsabilidad.
} 
Dentro de este marco conceptual, el presente trabajo tiene por objetivo explorar cómo las voces alternativas provenientes de una amplia gama de actores públicos dan lugar a la democracia urbana al transformar el espacio urbano en espacio público. Para lograr dicho objetivo, la primera sección busca explicar por qué varios críticos urbanos han dejado de lado las visiones pesimistas acerca del futuro urbano, y han asumido, por el contrario, una actitud característica del 'optimismo crítico'. Las siguientes secciones analizan cómo diferentes estrategias espaciales moldean la forma urbana, y cómo la democracia urbana puede surgir gracias a su interacción mutua. Finalmente, utilizo la noción de ciudad múltiple para discutir un modelo de urbanismo democrático, el cual se constituye de una diversidad de prácticas sociales y estrategias espaciales que son desplegadas por el 'público' urbano de manera cotidiana.

\section{2. ¿Es posible otra ciudad?}

Entre el inicio de la década de 1980 y la segunda mitad de la década de 1990, los científicos de la crítica social concordaban de cierta manera con la desaparición, o al menos con el encogimiento, de la esfera pública en las sociedades urbanas. Este proceso ha sido generalmente interpretado como el producto de fenómenos tales como la privatización y la fortificación del espacio social, los cuales han tenido lugar en ciudades norteamericanas y europeas bajo el triunfo de políticas conservadoras y la ideología neoliberal a partir de finales de la década de 1970 en adelante (Goheen, 1998; Banerjee, 2001).

A pesar de que la ciudad Occidental ha sido por largo tiempo objeto de "voces de decaimiento", tal como Robert Beauregard (1993) lo presenta con referencia a los Estados Unidos de postguerra, el punto inicial de esta última línea de 'pesimismo crítico' puede encontrarse en la mitad de la década de 1970. La crisis del modelo fordista-keynesiano de organización social se percibía como la vía de descomposición de la compleja herencia de uniones interpersonales y relaciones sociales sobre las que la vida pública urbana moderna se ha fundamentado. Como un precursor de este punto de vista, Richard Sennett (1974) en su cimentador estudio acerca de la 'caída del hombre público' ilustra cómo el triunfo del individualismo y la pérdida de interés por experiencias colectivas a partir del siglo XIx le quitan significado al dominio público urbano y lo dejan socialmente sin atractivo. 
Estas reflexiones fueron incursionadas por escritores interesados en el decaimiento del espacio público dentro de ciudades contemporáneas modernas. Los geógrafos urbanos y sociólogos, en particular, se han dado a la tarea de analizar cómo las tendencias de comodificación y militarización del espacio urbano han llevado a la formación de ciudades fortaleza y comunidades verjadas (Davis, 1990; Christopherson, 1994; Caldeira, 1996), a la emergencia de nuevas vías de desarrollo urbano desigual (Abu-Lughod, 1994; Smith, 1996) y, en general, a la "urbanización de injusticia" (Merrifield y Swyngedouw, 1996). Desde este punto de vista, y como consecuencia de la devaluación del terreno público en la ciudad postmoderna, la condición de ciudadanía urbana se ve inevitablemente reemplazada por el consumismo (Sorkin, 1992; Zukin, 1995; Amendola, 1997). Como una síntesis de estas perspectivas, Michael Dear y Steve Flusty (1998) han descrito la manifestación espacial de la ciudad postmoderna como una forma urbana sin centro caracterizada por la fragmentación y la especialización capitalista.

Este urbanismo postmoderno refleja el clima cultural en el cual el vuelco postmoderno ha ocurrido dentro de la teoría social vista como un todo. Esto ha sido profundamente inspirado por una peculiar forma de "milenarismo" (Jameson, 1990), que enfatiza la continua degeneración de la vida moderna social. Inclusive algunas importantes formulaciones del pensamiento crítico y radical no han podido escapar a esta actitud. Parecería, de hecho, que la pérdida de confianza en la acción colectiva y hasta individual en contra de la injusticia social no sólo ha distinguido al pensamiento en boga, sino también a muchos teóricos heterodoxos y radicales influidos por el estructuralismo o el postestructuralismo, como Marshall Berman (1982) lo ha sostenido en su impresionante crítica a Foucault. En la teoría sociológica de la postmodernidad o de la modernidad tardía, por ejemplo, uno puede encontrar indicios de esta actitud intelectual en lo que de otra manera serían fascinantes reseñas de las consecuencias de un nuevo sistema de reproducción social acerca de la vida personal en términos de un creciente "riesgo", "incertidumbre" y "corrosión del carácter” (Beck, 1992; Bauman, 1997; Sennett, 1998).

La respuesta a esta visión crítica, y finalmente pesimista, ha sido representada por aquellos quienes han promovido principios de mercado y auto-intereses económicos, y quienes han argumentado en favor de un modelo de revitalización urbana centrada alrededor de asociaciones público-privadas y de iniciativas 
en favor de las ganancias (Ashworth y Voogd, 1995; Porter, 1985). Una versión suave de esta postura centrada en el mercado ha sido presentada recientemente por representantes de la llamada 'nueva formulación liberal' (Harloe, 2001), en un intento por reconciliar "el éxito de mercado y la cohesión social" (Pahl, 2001). De esta manera, mientras en la década de 1980 y en la primera mitad de los años 1990, los enfoques dominantes de la Nueva Derecha con respecto a la política urbana, despreciaban deliberadamente los asuntos de justicia social e igualdad (Atkinson y Moon, 1994), la nueva dirección de las ideologías de democracia social en Europa Occidental ha marcado el camino hacia "el nuevo discurso de la competitividad, la cohesión social, la exclusión y el capital social" (Harloe, 2001: 889).

Como Michael Harloe lo señala, la nueva propuesta liberal para la política urbana ha surgido en "un periodo en el cual ha habido un cambio radical en la actitud para con las ciudades", que son ahora presentadas como "centros de competitividad, negocios, cultura, creatividad e innovación” (Harloe, 2001:889). Esta "nueva formulación liberal" ofrece grandes prospectos positivos para el futuro urbano, como lo enfatiza Ash Amin, Doreen Massey y Nigel Thrift en sus discusiones acerca de las políticas del partido New Labour en torno de las ciudades: "El reporte de Richard Rogers [1999] es maravillosamente entusiasta acerca de las ciudades y su potencial [...] En contra de la comúnmente aceptada visión distópica de las ciudades como fuentes de decaimiento económico, social y ambiental, al $90 \%$ de la población de Inglaterra que vive y trabaja en áreas urbanas se les promete un mejor futuro" (Amin, Massey, Thrift, 2000: 1-2).

Lo que hoy resulta novedoso es que tal ánimo positivo no sólo se encuentra articulado por los exponentes de la ideología dominante o por los reportes gubernamentales como el Reporte Rogers acerca del renacimiento urbano británico, sino también por un gran número de estudios urbanos críticos. En su excelente estudio de las nuevas direcciones de la teoría de planeación (la planeación comunicativa, el Nuevo Urbanismo y el modelo de ciudad justa), Susan Fainstein (2000) llega a la conclusión de que estas perspectivas, sin importar sus diferencias, "comparten un optimismo que ha estado faltando en las décadas previas" (Fainstein, 2000: 473). Dicho 'optimismo en resurrección' se combina con "el panorama reformista social” (Fainstein, 2000: 472), cuyo propósito es responder a los retos de la globalización y el postfordismo. 
Esto se vuelve más evidente en el ámbito de la ciudad justa cuyo optimismo ha sido revigorizado por la nueva postura del pensamiento utópico, aunque radicalmente pragmático, iniciado por eminentes representantes de la geografía neo-marxista y la teoría de planeación libertaria, tales como David Harvey (2000) y John Friedmann (2000), respectivamente. En su artículo, Fainstein (2000) correctamente señala que lo que unifica a la variedad de perspectivas acerca del asunto de la ciudad justa es el hecho de que los movimientos urbanos y la sociedad civil -en vez de las autoridades establecidas- son considerados como los referentes sociales privilegiados: "el propósito de su visión es el de movilizar un público en vez de prescribir una metodología a aquellos en la oficina" (Fainstein, 2000: 468).

En cualquier caso, el vínculo faltante en el análisis de Fainstein es aquél entre el cambio de actitud de los urbanistas críticos acerca de las ciudades y el escenario actual de la globalización. De hecho, mucho ha cambiado después del movimiento a nivel mundial de antiglobalización, el cual trajo (bajo la bandera de 'otro mundo es posible') la crítica radical al neoliberalismo y la afirmación de que un nuevo modelo de sociedad es posible y hasta necesario, hic et nunc. Más aún, las dificultades actuales del neoliberalismo provienen no sólo de acciones fundamentales de antiestablecimiento. Como Saskia Sassen lo indica, a partir de Arjun Appadurai (1996): "la globalización es un proceso que genera espacios contradictorios, caracterizados por la contestación. La diferenciación interna y el continuo cruce de fronteras" (Sassen, 1999: 193). Esto significa, desde mi punto de vista, que la relación dialéctica entre sociedades locales y el orden global se ha vuelto cada vez más problemática y multifacética. Efectivamente, en un gran número de regiones y ciudades, complejos procesos de cambio desde abajo y desde arriba han emergido en los últimos años, aumentando la gama de respuestas locales a la globalización. Tales vías hacia la "globalización desde abajo" (Magnaghi, 2000) han preparado el terreno para la formación de una visión pragmática-utópica de los futuros urbanos (Douglass y Friedmann, 1998; Fainstein, 1999; Holston, 1999).

Las secciones empíricas del presente artículo analizan una de estas respuestas locales a la globalización, la cual parece ser capaz de predecir un futuro de la justicia urbana y la democracia local, aunque frente a contradicciones persistentes y preguntas abiertas. La investigación considera el reciente cambio del centro histórico de Nápoles, el cual -sostengo- se convirtió, durante los años de la 
década de 1990, de un no-lugar a un espacio público (véase la fotografía 1). Mi tesis es que dicho cambio ha sido posible gracias a una compleja mezcla de cambios desde arriba (guiados por la nuevas élites políticas y el poder judicial local) y desde abajo (guiados por la sociedad civil activa y los movimientos sociales) (véase el cuadro 1). De esta manera, en esta parte empírica, describo primero la condición anterior del centro histórico de Nápoles como un no-lugar, y luego ilustro el proceso de cambio urbano que dicha área ha sufrido durante la última década

\section{Fotografía 1}

Piazza San Domenico como un no-espacio.

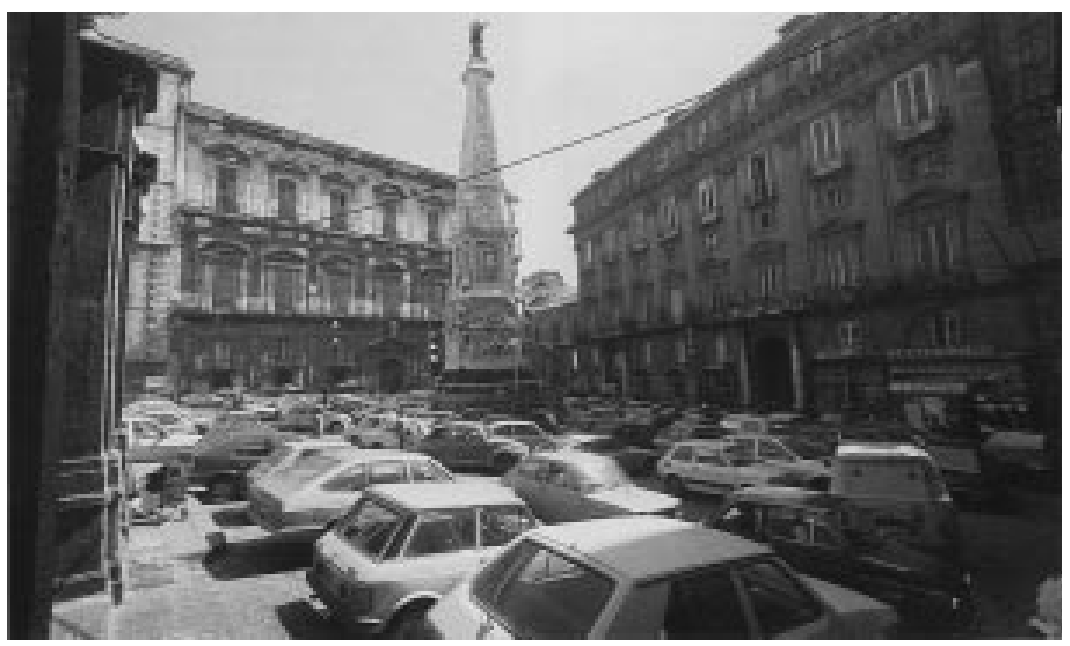

\section{Cuadro 1}

Democracia como multiplicidad de fuentes de poder social

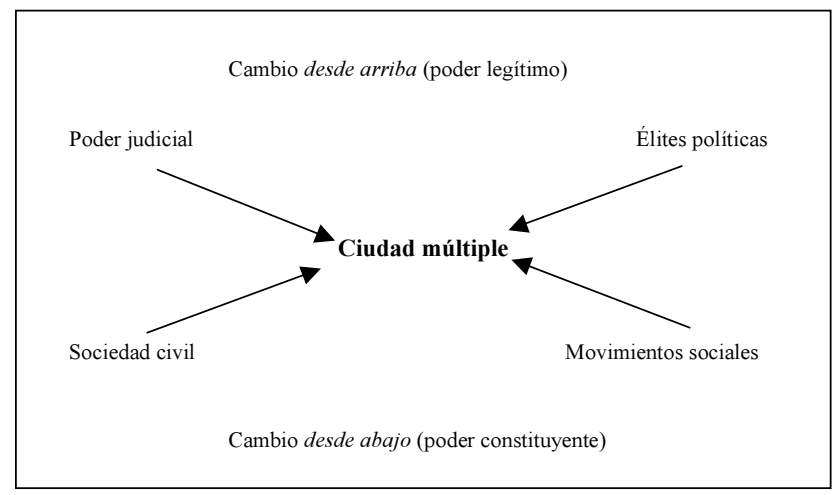

Fuente: Elaboración propia. 


\section{El centro histórico como un no-lugar}

Cuando Marc Augé (1992) habla acerca de los no-lugares, hace referencia a espacios que son el opuesto de los lugares, donde los científicos sociales son incapaces de descifrar la identidad de la gente, sus relaciones recíprocas y los símbolos de sus antecedentes históricos. Aunque Augé se refiere explícitamente a entidades sobremodernas y espacialmente limitadas (tales como autopistas, aeropuertos y supermercados), su noción de no-lugar puede ser metafóricamente aplicada a otras dimensiones geográficas o hasta a porciones enteras del espacio urbano. En esta sección argumento que este último es el caso del centro histórico de Nápoles hasta antes de la década de 1990. Durante las décadas de la posguerra, de hecho, esta área sufrió un proceso aparentemente irreversible de decaimiento físico, cultural y social, de tal suerte que la definición de no-lugar parece ser apropiada para referirse a su condición anterior.

A pesar de la rica herencia urbana (la cual incluye un vasto número de monumentos provenientes de varios periodos históricos, restos arqueológicos tanto subterráneos como en la superficie, una de las Universidades más antiguas de Europa y una añeja tradición de artesanías y comercialización de alta calidad) y a pesar de su inclinación natural como centro de la vida urbana en Nápoles, hasta la década de 1990 los usuarios del centro histórico estaban limitados esencialmente a sus habitantes. Lo que es más, durante las décadas de la Segunda Guerra Mundial, la mayoría de las calles del centro histórico fueron estranguladas por el tráfico durante el día, mientras que por la noche se encontraban iluminadas muy pobremente, desoladas e inhóspitas en general. Tanto así que las plazas de mayor valor histórico fueron usadas como estacionamientos para automóviles, y la mayoría de las iglesias eran permanentemente inaccesibles a los visitantes (véase el mapa I).

Dentro del marco conceptual de la metrópolis contemporánea, de acuerdo con Martinotti (1993), podemos definir este centro histórico como la 'primera generación' de espacio urbano en la manera que era usado primordialmente por sus propios habitantes (y, en menor grado, por otras personas tales como estudiantes y empleados de la Universidad, cuya presencia se encontraba escasamente integrada con el ambiente social local). 


\section{Mapa I}

Plazas confiscadas del centro histórico de Nápoles

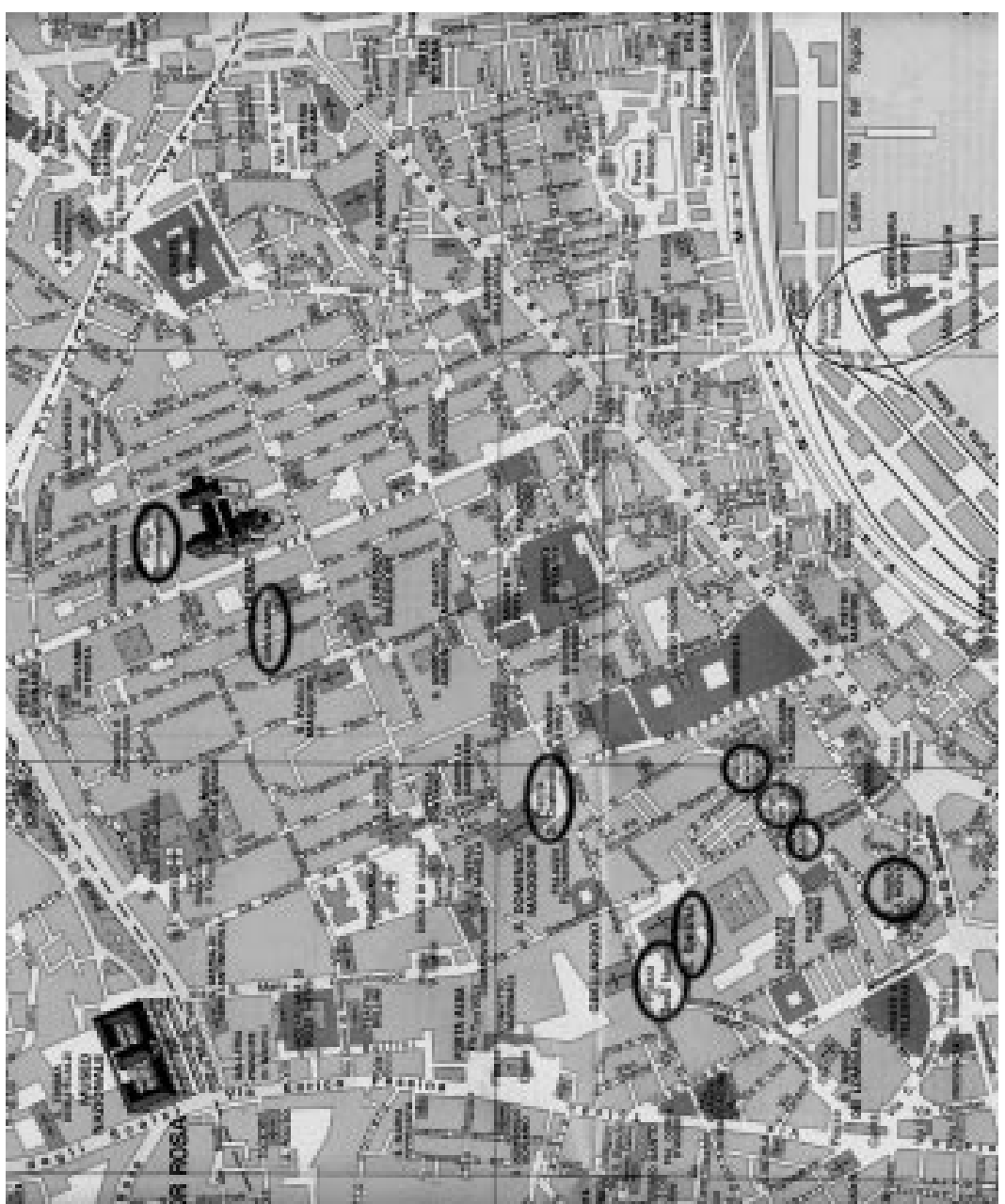


Además, un extendido decaimiento urbano despojó a la 'primera generación' del centro histórico de significado y le quitó todo atractivo social. Esto ocurrió en un contexto más amplio de decaimiento general que afectó a Nápoles después de la Segunda Guerra Mundial. En el transcurso de unas cuantas décadas, la crisis urbana llegó al grado de transformar a Nápoles en una suerte de "ciudad distópica" (Merrifield, 2000). No es sorprendente, entonces, el hecho de que un eminente historiador urbano napolitano haya descrito a la ciudad en los siguientes términos: "en un espacio de treinta años, una expansión caótica de edificios y una política industrial errónea han transformado a Nápoles en una de las ciudades más espantosas e inhabitables del mundo" (De Seta, 1977: 68).

De hecho, durante el periodo de posguerra la clase política gobernante marcó el camino para el decaimiento físico del ambiente urbano, usando al poder local para legitimar la expansión de la construcción y especulación (Dal Piaz, 1985; Belli, 1986). $\mathrm{Al}$ hacer esto, las clases gobernantes locales privaron a la sociedad civil de cualquier espacio de carácter público, donde los habitantes locales pudieran practicar y dar relevancia social a su ciudadanía urbana (Allum, 1973). Como resultado, los habitantes mismos percibían al espacio urbano como hostil y completamente ajeno a sus necesidades sociales.

En conclusión, el deterioro del ambiente construido, el decaimiento del espacio público y la crisis de la democracia urbana fueron fenómenos estrictamente interrelacionados en la Nápoles de posguerra. En este contexto, el centro histórico, a pesar de su papel como núcleo cultural de la ciudad, fue literalmente transfigurado por un mal gobierno local, y fue únicamente hasta la caída del poder de posguerra que pudo redescubrir su propia identidad de espacio público y social.

Las siguientes páginas tratarán acerca del proceso de cambio que ocurrió en este espacio a partir de la década de 1990. La siguiente sección toca el tema del cambio desde arriba, dirigido por la nueva mayoría política y el poder judicial local, mientras que la sección posterior habla del cambio urbano desde abajo, dirigido por una 'institucionalización' de la sociedad civil y los movimientos sociales urbanos. 


\subsection{El cambio urbano desde arriba: el centro histórico como lugar público}

Durante los primeros años de la década de 1990, Italia pasó por la transición de la Primera a la Segunda República. El régimen Cristiano-Demócrata que había gobernado Italia desde el final de la Segunda Guerra Mundial, sucumbió y finalmente se colapsó, seguido por una serie de investigaciones judiciales (conocidas como Tangentopoli o "Lugar de sobornos") que echaron luz sobre los cimientos ilegales de su sistema de poder.

En el vacío institucional que siguió al colapso de la Primera República, dos actores sociales hicieron su aparición en la escena pública, los cuales decían tener un papel en el proceso de transición: los jueces, cuyo rol fue legitimado por su vasta popularidad (Pizzorno, 1998), y un grupo de alcaldes de la ciudad (el llamado “movimiento de alcaldes": véase Vandelli, 1997), quienes tenían por objetivo inaugurar un nuevo estilo de gobierno local.

\subsubsection{El poder judicial}

En el debate general acerca de la transición italiana, muchos autores hablan acerca de la emergencia de un 'partido de jueces' para enfatizar la convergencia de intereses dentro del poder judicial. Aunque esta definición parece conceptualmente débil debido a muchos aspectos (por ejemplo, la falta de centralización de las decisiones políticas entre los jueces mina la idea de un 'partido' judicial específico), en términos empíricos puede resultar útil para acentuar el surgimiento de este nuevo actor público en Italia durante la década anterior (Bronzini, 1994). En otras palabras, puede decirse que una práctica italiana particular de "utilización de los procedimientos judiciales con fines políticos" (Ferrari Bravo, 1996) orientó la transición de la Primera a la Segunda República en los primeros años de la década de 1990.

Durante esta fase, la esfera de acción de los jueces dentro de la sociedad italiana se incrementó notablemente. Frecuentemente los jueces podían ejercer su influencia no sólo en la política nacional (siguiendo la operación Tangentopoli), sino también en la normatividad local (politicas locales), como ocurrió en el caso de Nápoles. En efecto, entre la primavera y el verano de 1993, a mediados de la transición política, un gran número de jueces de la Oficina de Acusación Pública (Fiscalía) ordenaron la confiscación de varias plazas históricas del centro. Al hacer esto, los jueces tenían en 
mente proteger dichos lugares contra automóviles y otras formas de ocupación ilegal del espacio público.

Al reclamar el carácter público de las plazas confiscadas, los jueces locales jugaron una parte decisiva en el inicio del proceso de renovación del centro histórico. Desde este punto de vista, los jueces asumieron efectivamente el papel de planificadores: una situación que puede ser definida como la utilización de los procedimientos judiciales con fines de normatividad.

La opinión pública local reaccionó escépticamente a estos eventos. Un planificador, por ejemplo, argumentó que "mientras el enfoque judicial de la planeación urbana podía ser legítimo en el caso de penas legales, no podía ofrecer soluciones reales a los problemas urbanos" (Discepolo, 1993). En retrospectiva, se tiene que reconocer la manera en que los jueces napolitanos lograron reabrir la cuestión de la revitalización del centro histórico que había sido despreciada por largo tiempo, principalmente debido a la inercia política e institucional y al mal gobierno.

Sin embargo, queda claro que otros actores, tanto institucionales como no institucionales, fueron capaces de poner en movimiento complejas formas de largo alcance para el re-descubrimiento y reapropiación del centro histórico. El primer actor fue indudablemente la nueva administración política, elegida en el gobierno de la ciudad a finales de 1993. Como mostraré en la siguiente sección, la administración de centro-izquierda, encabezada por el popular alcalde Antonio Bassolino, claramente abarcó las iniciativas de los jueces, legitimándolas con asuntos políticos. Es decir, las nuevas élites políticas pudieron construir un gran consenso de opinión alrededor de su acción, cuya falta había limitado inevitablemente el éxito de la intervención judicial en el centro histórico.

\subsubsection{Las nuevas élites políticas}

Después de la victoria electoral de 1993, el centro histórico se convirtió rápidamente en el foco simbólico de las estrategias de administración de la regeneración urbana (Bassolino et al., 1996). La renovación de áreas fue hecha de dos maneras: primero, a través de la implantación de políticas urbanas específicas; segundo, a través de la aprobación de un nuevo plan maestro que conformaba la Variante del Centro de la Ciudad, la cual incluía al centro histórico. 
Dentro de la primera, y de particular interés para este artículo, se encuentra la decisión del consejo de la ciudad a finales de 1994 de aprobar una resolución concerniente a la "renovación y adorno de varias áreas urbanas y plazas”. Claramente, este hecho enfatizó la continuidad de la política de la nueva administración con las medidas tomadas por los jueces para el centro histórico. Dada su declaración de insolvencia, la cual impuso restricciones prohibitivas en el presupuesto de la ciudad, el consejo ciudadano sugirió utilizar el dinero pagado por Alfredo Vito, un cristiano-demócrata ex miembro del parlamento local, como compensación por su participación en pagos ilegales y sobornos. La decisión tuvo un alto impacto simbólico entre la opinión pública puesto que en ese momento el caso de Vito encarnaba la degeneración del sistema político en el sur de Italia.

El dinero que Vito había devuelto a la administración de la ciudad fue dividido en varios proyectos, y un cuarto de la suma total fue asignada a la renovación y adorno de las plazas que habían sido confiscadas por la fiscalía. Debido a la limitada cantidad de recursos, fue únicamente posible intervenir en cuatro plazas, sin embargo el mismo documento se comprometía a completar la intervención tan pronto como el presupuesto de la ciudad pudiera costearlo. Eso es precisamente lo que ocurrió en los siguientes años. Hoy, todas esas plazas han sido renovadas y adornadas soberbiamente, y se han convertido en lugares centrales de encuentro para los ciudadanos locales y en atracciones turísticas populares (véase la fotografía 2).

Por otra parte, un rompimiento en la estrategia del gobierno local se pone en claro con la adopción del plan maestro durante la segunda mitad de la década de 1990. La Variante del Centro de la Ciudad (Comune di Napoli, 1999) extiende los límites de la vieja ciudad para incluir las áreas construidas después de la Segunda Guerra Mundial, aun aquellas que se encuentran localizadas fuera de lo que era reconocido como el centro de la ciudad. El objetivo de esta nueva delimitación espacial es el de conservar una mayor área de la ciudad en su forma urbana histórica (De Lucia, 1997; Gianni, 1995). El método de intervención está basado en los mismos criterios de clasificación topográfica y morfológica adoptados exitosamente a partir de la década de 1970 en ciudades tales como Boloña en Italia, Bamberg en Alemania, Amsterdam en Holanda y York en Gran Bretaña (Benevolo, 1993). El sistema de clasificación toma en cuenta tanto las construcciones como los lugares abiertos y está formulada considerando las par- 


\section{Fotografía 2}

Piazza San Domenico después del proceso de renovación

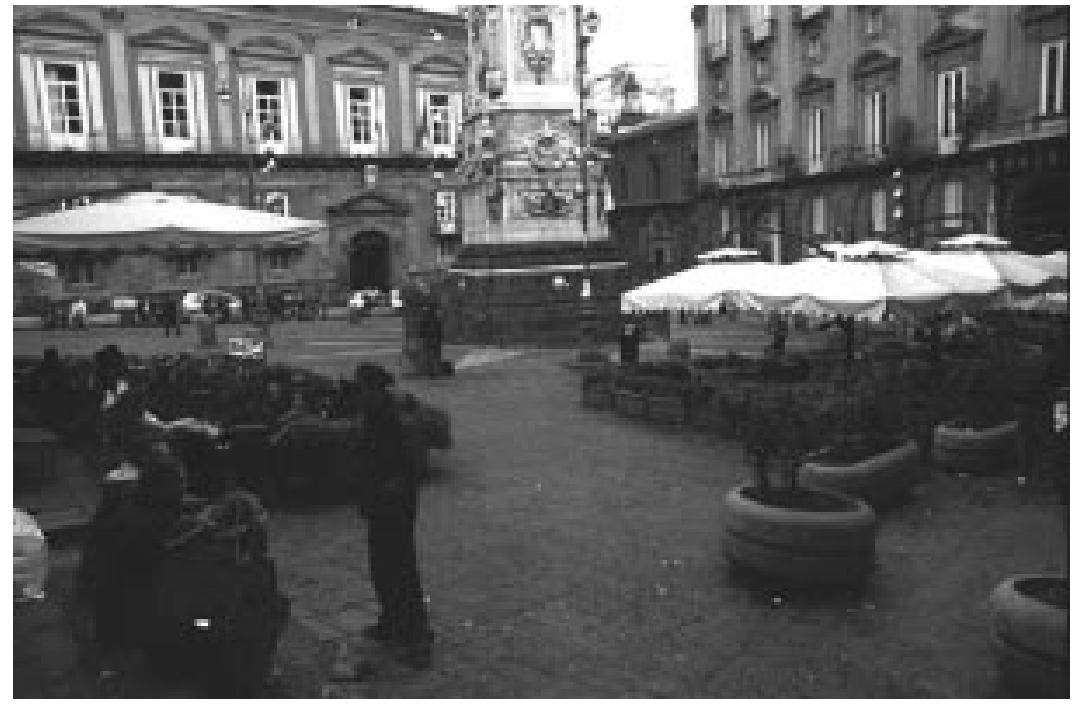

tes constitutivas de la estructura urbana y los procesos a largo plazo de la transformación histórica para identificar formas urbanas recurrentes.

De acuerdo con el director del departamento de planeación de la ciudad, el nuevo plan maestro refleja una nueva forma de "planear austeridad" la cual busca "proteger la integridad física y rehabilitar la identidad cultural de la ciudad" (Gianni, 1997: 100). El planificador urbano Giuseppe Campos Venuti observó que el plan era un ejemplo de "política de planeación reformista", la cual "está consciente de la presencia y necesidades del mercado, pero al mismo tiempo regula al mercado mismo para garantizar los intereses de la comunidad local, prevenir la especulación de construcción y estimular las actividades empresariales" (Campos Venuti, 1997: 100).

Desde este punto de vista, es importante subrayar la "perspectiva reformista social” que inspira al proceso de planeación. Por sí misma, es evidentemente incapaz de resolver las contradicciones intrínsecas al proceso de planeación de la ciudad bajo el modo de desarrollo capitalista (Harvey, 1989), como se ve reflejado por el número de problemas sociales que aún afecta a Nápoles, tales como la persistente alta tasa de desempleo (alrededor de $40 \%$ de la población activa). Sin embargo, la nueva dirección en el gobierno local ha logrado una mejora en la calidad del ambiente urbano 
construido del centro histórico y de la ciudad en general, dando lugar a una mayor perspectiva positiva sobre el futuro de Nápoles.

\subsection{Cambio urbano desde abajo: el papel de la sociedad civil}

Existen dos tipos de actores sociales que han basado sus acciones en el centro histórico: en primer lugar, aquellos que representan lo que podría definirse como sociedad civil institucionalizada, la cual se caracteriza por la búsqueda de soportes concretos en las instituciones públicas; en segundo lugar, los movimientos sociales urbanos, los cuales por el contrario tienen con frecuencia relaciones en conflicto con las autoridades establecidas, formulan cuestiones radicales acerca de la democracia y destacan visiones antisistémicas del cambio urbano.

Me parece que es importante enfatizar las diferencias entre estos dos tipos de actores sociales: por un lado, de hecho difieren con respecto a sus estrategias, ideologías y connotaciones sociales; pero, por otro lado, comparten un rasgo básico en la manera en la que ambos intentan tomar acciones en el espacio urbano para transformarlo en espacio público. Dentro de mi marco teórico, estos actores representan las fuentes de 'poder constituyente' dentro de las ciudades en tanto que dan forma a las visiones desde abajo de los procesos de cambio urbano.

\subsubsection{La sociedad civil institucionalizada}

A pesar de algunos reportes por parte de las autoridades que presentan al sur de Italia como falto de espíritu cívico y público (Putnam, 1993), los fenómenos asociativos y, en general, las formas de movilización cívica han estado presentes en todo la sociedad sureña en el transcurso de las últimas décadas (Ramella, 1995; Piperno, 1997; Alcaro, 1999).

En particular, a partir de los primeros años de la década de 1990, el colapso del régimen Cristiano-Demócrata puso fin a la densa red de relaciones de poder que anteriormente habían permeado varios sectores de la sociedad civil en el sur de Italia, evitando que desarrollaran estrategias sociales autónomas. En la escala urbana, esto significó un estado de crisis para aquellos fenómenos asociativos que estaban estrechamente relacionados con el sistema político. Si en el pasado la sociedad sureña mostraba una actitud mucho más débil hacia la participación social y la 
movilización cívica, era precisamente debido al funcionamiento de dicha red de relaciones de poder y no a la pretendida presencia de algunas características distintivas dentro de la mentalidad sureña y el sistema cultural, como Robert Putnam lo sostiene (con respecto a la participación cívica, véase también Putnam, 1993 y el ensayo crítico de Short, 2001).

En Nápoles, la caída de la Primera República y las nuevas relaciones entre las instituciones públicas y los ciudadanos locales abrieron nuevos espacios de acción para las sociedades civiles 'organizadas'. El caso del centro histórico de Nápoles ejemplifica perfectamente las nuevas tendencias de asociación socio-institucional. De particular interés fueron las campañas promovidas por la Fondazione Napoli 99, que alentó el redescubrimiento de la herencia cultural y artística del centro histórico de la ciudad.

La primera campaña de Napoli 99 tenía por objetivo promover cierto tipo de vinculación entre estudiantes y monumentos del centro histórico ("cada escuela adopta un monumento" como su frase publicitaria lo expresaba), a través de asociaciones con las instituciones públicas tales como las varias Superintendencias o Soprintendenze (para los Bienes Históricos, para los Bienes Arquitectónicos y Ambientales, y para los Bienes Arqueológicos) y la autoridad educativa, y a través de apoyos del Ministerio de Educación y el Ministerio para los Bienes Culturales y Ambientales.

En 1992, Napoli 99 organizó otra campaña, estrechamente vinculada con la anterior, llamada Monumenti Porte Aperte o 'Monumentos a Puerta Abierta'. Su propósito era abrir varios monumentos del centro histórico que generalmente se encontraban cerrados al público. Como resultado, un buen número de estos monumentos fue restaurado y hoy se encuentran permanentemente abiertos y accesibles.

Varios autores están de acuerdo acerca del preponderante papel que estas campañas han tenido en la revitalización del centro histórico de la ciudad. Para aquellos que señalan la relevancia de la relación entre los habitantes locales y la herencia urbana, estas campañas son consideradas como pasos decisivos hacia la reapropiación simbólica de la historia urbana y la cultura como parte integral de los ciudadanos napolitanos, e hicieron contribuciones significativas para la mejora de la imagen pública de la ciudad (Governa, 1997: 147-180; Cattedra y Memoli, 2000).

En conclusión, uno podría considerar estas campañas como símbolos de una utopía burguesa de la ciudad, la cual pone énfa- 
sis en la primacía de la identidad cultural en la vida urbana cotidiana. Como lo mostraré en la siguiente sección, esto difiere profundamente del radicalismo de la otra fuente de poder constituyente en las ciudades: los movimientos sociales urbanos.

\subsubsection{Los movimientos sociales urbanos}

Durante la década de 1990, algunos movimientos sociales locales enfocaron su acción en el centro histórico. Esto se debía principalmente a la presencia de los estudiantes, quienes tenían un papel decisivo en las movilizaciones políticas y sociales desde el movimiento de 1968. Después de un largo hiato durante la década de 1980, los años 90 vieron un resurgimiento de los movimientos estudiantiles tanto en Nápoles como en el resto de Italia. En 1990, los departamentos de la Universidad fueron ocupados por un plazo de tres meses por estudiantes que protestaban en contra del proyecto del gobierno con respecto a la autonomía financiera de las Universidades, lo cual era considerado como un primer paso hacia la privatización de la educación pública. Un segundo movimiento de acción estudiantil ocurrió en 1994 con la protesta en contra del aumento a colegiaturas.

Aunque las movilizaciones estudiantiles estaban interesadas principalmente en asuntos meramente universitarios, su papel en el centro histórico durante la década anterior ha sido relevante esencialmente por dos razones: la primera, las varias ocupaciones estudiantiles de los departamentos llevó a un redescubrimiento colectivo del centro histórico como lugar de socialización y de organización de eventos. Este fenómeno se desarrolló a tal grado, que para la segunda mitad de la década de 1990, el centro histórico pronto se convirtió en uno de los lugares principales de entretenimiento en Nápoles.

La segunda razón es que para continuar con sus actividades sociales y cívicas, muchos estudiantes que participaron en la ocupación de la Universidad crearon nuevos centri sociali ('centros sociales'), estableciendo una presencia estable de los movimientos sociales urbanos en el centro histórico.

Es necesario enfatizar el primer punto, puesto que la identidad del centro histórico como lugar de consumo parece estar volviéndose cada vez más penetrante. En el transcurso de unos cuantos años, un gran número de pequeños restaurantes de comida rápida (la mayoría de propietarios locales) y otros tipos de tiendas han abierto sus puertas en el centro histórico, mientras que cier- 
tas calles se encuentran literalmente abarrotadas con bares y discotecas. Aunque grandes 'empresas de entretenimiento' no parecen operar dentro del área, la pregunta acerca de si el centro histórico se ha convertido en un 'distrito de placer' (Bonomi, 2000) de la misma manera que ha sucedido en otras ciudades, es digna de atención. Los activistas urbanos son bastante críticos en este punto: dos miembros de un comité estudiantil ven este proceso en términos de "comercialización y normalización del centro histórico, que está llevando a una degeneración partiendo de un lugar de movilización política y social-como lo era en los primeros años de la década de 1990- a un efímero lugar de entretenimiento -como ocurre actualmente" (comunicación personal, junio de 2001).

Esta cuestión resulta relevante en vista de que los movimientos radicales actuales no sólo están comprometidos con la promoción de acciones políticas, como era el caso en la década de 1970, sino también con el patrocinio de una sociabilidad alternativa en el ámbito público (Ruggiero, 2000). Esto introduce al segundo punto en la discusión: la continuidad del activismo joven en la década de 1990 dentro de los centri sociali. Después del movimiento de 1994, grupos estudiantiles y de activistas urbanos ocuparon algunos de los edificios abandonados del centro histórico, revirtiendo una vieja estrategia de creación de centros sociales en áreas alejadas del centro histórico, ya sea en las afueras o en los suburbios de las áreas urbanas.

Entre estos nuevos centros sociales, vale la pena considerar el caso del Laboratorio Ocupato SKA, cuya característica específica es su localización en una calle muy céntrica y visible del centro histórico. Desde sus inicios, esta posición estratégica hizo que los activistas pensaran en SKA como el 'cuartel de comunicaciones' ideal de los movimientos sociales de la ciudad, y como una base para las formas independientes de información y contrainformación. Una dimensión espacial dual -interna/externa- fue la práctica de los activistas del centro social: por una parte, promovían una amplia gama de actividades dentro del centro social (tales como la organización de una clínica para migrantes y un centro de cómputo gratuito), y por la otra, organizaban campañas para la reevaluación de varios espacios degradados del centro histórico con el fin de transformarlos en espacios públicos.

En pocas palabras, se intentaba resaltar modelos extrainstitucionales de la esfera pública y la democracia (Collettivo, 'Luogo Comune', 1992) con el hilo conductor de otros centros sociales 
italianos (Consorzio Aaster et al., 1996). Desde este punto de vista, los centros sociales representan la construcción de un peculiar tipo de esfera pública, la cual es tanto un espacio público de debate en toda la ciudad y un lugar de acción en el ambiente urbano que la rodea (Vecchi, 1994).

\section{Conclusiones}

En este artículo he buscado interpretar la formación del espacio público de Nápoles durante la década de 1990 que tuvo lugar como resultado de la interacción entre procesos dinámicos de cambio institucional local y el surgimiento de demandas fundamentales para el cambio urbano. Este proceso ha sido analizado desarrollando la idea de una ciudad múltiple en el contexto de Nápoles, para enfatizar la variedad de fuentes de poder social que nutren a la democracia urbana.

Como se explica en la introducción, el asunto de la democracia ha vuelto a ser puesto en boga dentro de la agenda de la investigación urbana como consecuencia del renovado optimismo que hoy alenta a la comunidad científica. Sin embargo, lo que todavía queda sin ser contestado dentro de la literatura general es la idea de la democracia urbana misma. Mi investigación ha querido ir más allá de la idea generalizada de que las estrategias urbanas tienden a converger hacia un punto de estabilidad en la búsqueda de soluciones consensuales. Por el contrario, he tratado de comprender la complejidad de los procesos contemporáneos del cambio urbano presentando un marco de análisis en dos niveles: desde arriba y desde abajo. El primero se centra en los actores que poseen el 'poder legítimo' sobre las ciudades, mientras que el segundo pone atención en la acción de un conjunto de contrafuerzas urbanas que personifican al "poder constituyente" en el ámbito público (Hardt y Negri, 2000).

Interpretado de esta manera, el enfoque de la ciudad múltiple nos lleva a reconsiderar la idea de espacio público en la ciudad democrática, tomando en cuenta no solamente la esfera pública 'oficial', que sigue los caminos convencionales de la legitimación institucional, como es el caso de las plazas confiscadas del centro histórico de Nápoles, sino también otros espacios de democracia no institucionalizada que se encuentran más allá del Estado y de las políticas representativas (Amin, Massey y Thrift, 2000), como es el caso de los centros sociales. Subrayando la coexistencia de fuentes alternativas de poder como condición esencial de la de- 
mocracia urbana, tal visión del ámbito público ofrece la posibilidad de evitar la alternativa 'ciega' entre conflicto y cooperación que limita el desarrollo de las relaciones dinámicas entre el poder legítimo y el poder constituyente en las ciudades contemporáneas.

\section{Bibliografía}

Abu-Lughod, J. L., ed. (1994), From urban villane to east village, Blackwell, Oxford.

Alcaro, M. (1999), Sull'identità meridionale. Forme di una cultura mediterránea, Bollati-Boringhieri, Turín.

Allum, P. A. (1973), Politics and society in post-war Naples, Cambridge University Press, Cambridge (UK).

Amendola, G. (1997), La città posmoderna. Magie e paure della metropoli contemporanea, Laterza, Roma y Bari.

Amin, A. y S. Graham (1997), “The ordinary city”, Transactional Institute of British Geographers, vol. 22, 411-429.

Amin, A., Massey, D. y N. Thrift (2000), Cities for the many not the few, Policy Press, Bristol.

Amin, A. y N. Thrift (2002), Cities. Reimagining the urban, Polity Press, Cambridge (UK).

Appadurai, A. (1996), Modernity at large: Cultural dimensions of globalization, University of Minesota Press, Minneapolis.

Ashworth, G. J. Y H. Voogd (1995), Selling the city: marketing approaches in public sector urban planning, John Wiley, Chichester.

Atkinson, R. y G. Moon (1994), Urban policy in Britain. The city, the state and the market, MacMillan, Houndmills Basingstoke.

Augé, M. (1992), Non-lieux. Introduction à une anthropologie de la surmodernitè, Seuil, París. 
Banerjee, T. (2001), “The future of public space. Beyond invented streets and reinvented places", Journal of American Planning Association, vol. 67, núm. 1, 9-24.

Bassolino, A. et al. (1996), Verso un rinascimento napoletano, Liguori, Nápoles.

Baumann, Z. (1997), Postmodernity and its discontents, Blackwell, Oxford.

Beauregard, R. A. (1993), Voices of decline. The postwar fate of us cities, Blackwell, Oxford.

- y S. Body-Gendrot, eds. (1999), The urban moment cosmopolitan essays on the late-20 $0^{\text {th }}$-Century city, Sage, Thousand Oaks.

Beck, U. (1992), Risk society. Toward a new modernity, Sage, Londres.

Belli, A. (1986), Il labirinto e l'eresia. La politica urbanistica a Napoli tra emergenza e ingovernabilità, Angeli, Milán.

Benevolo, L. (1993), The European city, Blackwell, Oxford.

Berman, M. (1982), All that is solid melts into air. The experience of modernity, Simon \& Schuster, Nueva York.

Bonomi, A. (2000), Il distretto del piacere, Bollati-Boringhieri, Turín.

Bronzini, G., ed. (1994), Il potere dei giudici, Manifestolibri, Roma.

Caldeira, T. (1996), "Fortified enclaves: The new urban segregation", Public Culture, núm. 8, 303-328.

Campos Venuti, G. (1997), "Napoli e l'urbanistica riformista", Urbanistica, núm. 109, 100-105.

Cattedra, R. y M. Memoli (2000), "La reappropriation du patrimoine symbolique du centre historique de Naples", me- 
meo, Institut des Hautes Études del'Amérique Latine, París.

Christopherson, S. (1994), "The fortress city: Privatized spaces, consumer citizenship", en A. Amin, ed., Post-Fordism. A reader, Blackwell, Oxford (UK) and Cambridge (MA).

Collettivo 'Luogo Comune' (1992), “Per una democrazia extraparlamentare", Derive Approdi, núm. 0, 49-51.

Comune di Napoli (1999), Variante al piano regolatore generale. Centro storico, zona orientale, zona noroccidentale, Assessorato alla Vivilità, Nápoles.

Consorzio Aaster et al. (1996), Centri sociali: Geografie del desiderio, Shake, Milán.

Dal Piaz, A. (1985), Napoli 1945-1985. Quarant'anni di urbanistica, Angeli, Milán.

Davis, M. (1990), City of quartz. Excavating the future of Los Angeles, Verso, Londres.

Dear, M. y S. Flusty (1998), "Postmodern urbanism", Annals of the Association of American Geographers, vol. 88, núm. 1, 50-72.

De Lucia, V. (1997), "Il processo di pianificazione a Napoli. La ripresa di un discorso interrotto”. Urbanistica, núm. 109, 91-111.

Dematteis, G., Indovina, F., Magnaghi, A., Piroddi, E., Scandurra, E. y B. Secchi (1999), I futuri della città. Tesi a confronto, Angeli, Milán.

De Seta, C. (1977), Città, territorio e mezzogiorno in Italia, Einaudi, Turín.

Discepolo, B. (1993), “La via giudiziaria all'urbanistica”, Ter., núm. 3, p. 2. 
Douglass, M. y J, Friedmann, eds. (1998), Cities for citizens: Planning and the rise of civil society in a global age, John Wiley, Chichester.

Fainstein, S. S. (1999), “Can we make the city we want?”, en R. A. Beauregard y S. Body-Gendrot, eds., The urban moment. Cosmopolitan essays on the late-20 $0^{\text {th }}$-Century city, Sage, Thousand Oaks.

- (2000), "New directions in planning theory", Urban Affairs Review, vol. 35, núm. 4, 451-478.

Ferrari Bravo, L. (1996), "Processo all'italiana”, Futuro Anteriore, núm. 5, 120-145.

Friedmann, J. (2000), “The good city: In defense of utopian thinking”, International Journal of Urban and Regional Research, vol. 24, núm. 2, 460-472.

García, S. (1996), “Cities and citizenship”, International Journal of Urban and Regional Research, vol. 20, núm. 1, 7-21.

Gianni, R. (1995), "Il centro storico e le aree ex industriali”, Spazio e Società, núm. 69, 67-72.

__ (1997), “Un'urbanistica austera”, Urbanistica, núm. 109, 99-110.

Goheen, P. (1998), "Public space and geography of the modern city", Progress in Human Geography, vol. 22, núm. 4, 479496.

Governa, F. (1997), "Napoli: la riconquista del passato nella construzione del presente”, en Id., Il milieu urbano. L'identità territoriale nei processi di sviluppo, Angeli, Milán.

Harloe, M. (2001), "Social justice and the city: The new 'liberal formulation", International Journal of Urban and Regional Research, vol. 25, núm. 4, 889-897.

Hardt, M. y A. Negri (2000), Empire, Harvard University Press, Cambridge (MA). 
Harvey, D. (1989), The urban experience, Blackwell, Oxford.

- (2000), Spaces of hope, Edinburgh University Press, Edimburgo.

Holston, J., ed. (1999), Cities and citizenship, Duke University Press, Durham (NC).

Imrie, R., Pinch, S. y M. Boyle (1996), "Identities, citizenship and power in the cities", Urban studies, vol. 33, núm. 8, $1255-1261$.

Jameson, F. (1990), Postmodernism, or the cultural logic of late capitalism, Duke University Press, Durham (NC).

Mann, M. (1993), The sources of social power. The rise of classes and nation-states, 1970-1914, vol 2. Cambridge University Press, Cambridge (UK).

Magnaghi, A. (2000), Il progetto locale, Bollati Boringhieri, Turín.

Martinotti, G. (1993), Metropoli. La nouva morfologia sociale della città, Il Mulino, Boloña.

Merrifield, A. (2000), "The dialectics of dystopia: Disorder and zero tolerance in the city", International Journal of Urban and Regional Research, vol.24, núm. 2, 473-390.

Merrifield, A. y E. Swyngedouw, eds. (1996), The urbanization of injustice, Lawrence \& Wishart, Londres.

Pahl, R. (2001), "Market success and social cohesion”, International Journal of Urban and Regional Research, vol. 25, núm. 4, 879-883.

Piperno, F. (1997), Elogio dello spirito pubblico meridionale: genius loci e individuo sociale, Manifestolibri, Roma.

Pizzorno, A. (1998), Il potere dei giudici. Stato democratico e controllo della virtù, Laterza, Roma y Bari. 
Porter, M. (1985), “The competitive advantage of the inner city”, Harvard Business Review (reimpresa en R. T. LeGates y F. Stout, eds., 2000. The city reader, Routledge, Londres y Nueva York).

Putnam, R. (1993) Making democracy work. Civic traditions in modern Italy, Princeton University Press, Princeton.

Ramella, F. (1995), "Mobilitazione pubblica e società civile meridionale”, Meridiana, núm. 22-23, 121-154.

Ruggiero, V. (2000), Movimenti nella città. Gruppi in conflitto nella metropoli europea, Bollati-Boringhieri, Turín.

Sassen, S. (1999), "Whose city is it?" Globalization and the formation of new claims", en J. Holston, ed., Cities and citizenship, Duke University Press, Durham (NC).

Sennett, R. (1974), The fall of public man, Cambridge University Press, Cambridge (UK).

- (1998), The corrosion of charachter. The personal consequences of work in the new capitalism, W. W. Norton \& Company, Nueva York y Londres.

Short, J. R. (2001), “Civic engagement and urban America”, City, vol. 5, núm. 3, 271-280.

Smith, N. (1996), The new urban frontier: Gentrification and the revanchist city, Routledge, Londres y Nueva York.

Sorkin, M., ed. (1992), Variations on the theme park: The new American city and the end of public space, Hill and Wang, Nueva York.

Thrift, N. (2001) "How should we think about place in a globalising world?", en A. Madanipour, A. Hull y P. Healey, eds., The governance of place. Space and Planning Processes, Ashgate, Aldershot.

Vandelli, L. (1997), Sindaci e miti, Il Mulino, Boloña. 
Vecchi, B. (1994), "Frammenti di una diversa sfera pubblica”, en vv. AA., Comunità virtuali. I centri sociali in Italia, Manifestolibri, Roma.

Zukin, S. (1995), The culture of cities, Blackwell, Cambridge (MA).

Enviado: 27 de septiembre de 2002. Aceptado: 22 de enero de 2003. 